\title{
Report of the Coding Committee of the Association for European Paediatric Cardiology
}

\author{
Rodney C. G. Franklin, ${ }^{1}$ Robert H. Anderson, ${ }^{1}$ Otto Daniëls, ${ }^{2}$ Martin J. Elliott, ${ }^{1}$ Marc H. M. L. Gewillig, ${ }^{3}$ \\ Renzo Ghisla, ${ }^{4}$ Otto N. Krogmann, ${ }^{5}$ Herbert E. Ulmer ${ }^{6}$ and Franco P. Stocker ${ }^{7}$ \\ ${ }^{1}$ London, UK; ${ }^{2}$ Nejmegen, The Netherlands; ${ }^{3}$ Leuven, Belgium; ${ }^{4}$ Zurich, Switzerland; ${ }^{5}$ Duisburg, Germany; \\ ${ }^{6}$ Heidelberg, Germany; ${ }^{7}$ Chairman, Bern, Switzerland
}

$\mathrm{I}$ N 1999 AND EARLY 2000, THE ASSOCIATION FOR European Paediatric Cardiology published the European Paediatric Cardiac Code as independent but linked Short and Long Lists, containing 650 and 3876 primary terms respectively. ${ }^{1,2}$ The historical background and rationale for development of this coding system has been previously detailed, but essentially it followed a series of meeting of the coding committee of the Association between 1997-1999, during which a pre-existing Long List was adopted and then used to create the condensed Short List. ${ }^{1}$ The system was published as the recommended standard coding system for use across Europe, covering the diagnosis and therapy of children with congenital and acquired cardiac disease. The scope of the lists was to encompass the needs of all those involved with such patients, from the fetal cardiologist through to the specialist in adult congenital heart disease; and from the general paediatric cardiologist and cardiac surgeon, to those specialising in transcatheter interventions, paediatric electrophysiology, and paediatric echocardiographers. In addition, the code was crossmapped to the 9 th and 10 th revisions of the International Classification of Diseases ("ICD-9" and "ICD-10") provided by the World Health Organisation in order to facilitate returns to central government, a requirement in most countries. In so doing, it was hoped to address the concerns of many centres that such information submitted by professional coding staff was often inaccurate due to the complex nature of congenital cardiac disease, together with the limited scope and vague terminology of the International listings.

Correspondence to: Dr Rodney CG Franklin, Paediatric Cardiologist, Harefield Site, Royal Brompton and Harefield NHS Trust, Harefield, Middlesex UB9 6JH, UK. Tel/fax: 01895 828659; E-mail: r.franklin@rbh.nthames.nhs.uk
The Long List was designed as a fully comprehensive detailed hierarchical system, for use by those requiring enhanced detail for research and audit, as well as for eventual incorporation as part of the electronic patient record. In contrast, the Short List was created specifically to be part of a database for auditing the workload of the paediatric cardiac specialist, whether working in an individual centre or as part of multicentric national, or international co-operative studies. It was intended for day-to-day use by the clinician, for whom a more detailed diagnostic and therapeutic breakdown was not necessary, or deemed to be too time-consuming. The Short List was designed to remain comprehensive by inclusion of "collective terms", so that the user could cover all eventualities without the need for the longer list. The two lists would not be interdependent, although easily useable together, with appropriate software, as a two level system.

The requirement to undertake inter-institutional audit is, if anything, even more pressing now than at the time of first issuing the European Code, as demonstrated by the publication of the inquiry into the outcome of paediatric cardiac surgery at the centre in Bristol, in the United Kingdom. ${ }^{3,4}$ Subsequent analysis of data submitted to, and commissioned by, this inquiry permitted the drawing of possible conclusions, such as lower mortality correlating with a higher workload of surgical cases, yet also produced criticism of the methods of gathering such data. ${ }^{5,6}$ The use of different crude coding schemes, such as the 9th and 10th revisions of the International Classification of Diseases, which fail adequately to deal with the issues of case-mix, as well as poor, if any, validation of the collected data, indicates that any conclusions drawn from such studies must be tentative at best. There is now an obligation in many countries, driven 
both by government and peer-group pressure, to provide national and international comparisons of surgical results between centres caring for children with heart disease. Significantly, there is also widespread agreement that such comparisons of outcomes must include meaningful stratification of risk when analysing such data, and that the data itself must be accurate morphologically and be validated. ${ }^{6,7}$ This need is exemplified in paediatric cardiology by the inherent broad spectrum of congenital cardiac lesions, and the varying health of the individuals when presenting for therapeutic interventions. These factors may then be linked to others, such as whether the lesion has been antenatally diagnosed. ${ }^{8}$ Although there is now increasing recognition by governments of the importance of such processes of audit, the underfunding of these initiatives, both technologically and in terms of human resources, remains a major source of concern and a hindrance to progress.

Since its publication, the European Paediatric Cardiac Code has been accepted increasingly throughout Europe. It is presently used by between 50 and 60 centres for paediatric cardiology within Europe. In Germany, the Short List is used within the government-led programme of National Quality Control, involving both paediatric cardiologists and cardiac surgeons. In Switzerland, the Swiss Society of Paediatric Cardiology is using the Short List in a national audit project. Other multicentric national studies are planned in the near future in the Netherlands and Belgium. In the United Kingdom, it is now obligatory for each paediatric cardiac centre to submit diagnostic and procedural data on all patients with congenital cardiac lesions undergoing interventions. The data is sent to a central body, the United Kingdom Paediatric Central Cardiac Audit Database, which then independently analyses the data to produce outcomes specific to each centre. As from April 2003, it will be necessary for the centres to submit their returns as coded using the Short List of the European Paediatric Cardiac Code.

The European Paediatric Cardiac Code has now been published on the website of the Association for European Paediatric Cardiology (www.aepc.org/codecom.htm) for free down load, conditional upon agreement not to alter the system, and to respect the copyright of the Association. To date, 340 individuals or groups have accessed the system in this way, not only from Europe, but also from other continents, confirming the wide interest in the code. At present, there are also versions translated to the German and Dutch languages which are available on the website.

The coding committee has always recognised the need for regular revisions of the code so as to encompass changes in clinical practice, and to address deficiencies identified by users and fed back to the committee. Other users have submitted suggestions for improvement. In addition, there was also the need formally to crossmap the Long List to the Short List, so that those using both Lists could easily transfer data from one to the other, thus enabling submission of data to studies using the Short List, or for central returns. The articles contained in the supplement to this fascicle of the Journal addresses these issues. In addition, the supplement provides a bidirectional crossmap between the Short Lists of the European Paediatric Cardiac Code and the Congenital Heart Surgery Nomenclature and Database Project. The surgical list was published shortly after the European code, ${ }^{9}$ and it is pertinent to give a brief review of its history.

\section{The International Congenital Heart Surgery Nomenclature and Database Project}

A few months after the European Paediatric Cardiac Code was published in this Journal, ${ }^{2}$ the International Congenital Heart Surgery Nomenclature and Database Project published its report. ${ }^{9}$ It was endorsed by the Congenital Heart Surgery Committees of the Society of Thoracic Surgeons and the European Association for Cardiothoracic Surgery, as well as the European Congenital Heart Surgeons Foundation. As with the European code, the surgical system has a comprehensive Long List, and a condensed, or "minimal", data set. Although there is a similar underlying aim of the two systems, namely to establish a standardised nomenclature for congenital cardiac disease, the overriding drive for the more surgical publication was to provide a system which would be the basis for collection of surgical data, so as to enable multicentric and international comparisons of surgical outcomes with case-mix and stratification of risk. To this end, the minimal data set was produced, with 25 mandatory and 13 optional fields of entry. This uses a Short List of diagnostic and surgical procedural terms, derived from their comprehensive data set, and divided into four subcategories: Non-cardiac diagnoses and general pre-operative risk factors; Diagnoses; Procedures; and Complications. There are no numerical codes attached to the terms. This minimal dataset has recently been revised, with proposals for a major international co-operative harvest of data. ${ }^{10}$ It is available for free download from the web (www.ctsnet.org/doc/5357; www.pediatric.ecsur.org).

\section{Crossmap between the European Paediatric Cardiac Code and the International Congenital Heart Surgery Nomenclature and Database Project}

As a result of these near simultaneous publications, representatives of those involved with the two systems 
from the Association for European Paediatric Cardiology, the Society of Thoracic Surgeons, and the European Association for Cardiothoracic Surgery agreed in October 2000 that the three societies should work in partnership and produce a reconciliatory bidirectional map between the two systems. ${ }^{11}$ The feasibility for the interaction was established initially by creating a crossmap between the two Short Lists. The process was subsequently approved by the 250 participants at the First International Summit on Nomenclature for Congenital Heart Disease on 27 May, 2001, held at a designated session of the Third World Congress of Paediatric Cardiology and Cardiac Surgery in Toronto, Canada. The crossmap of the Short Lists was approved, along with the aim in the longer term of crossmapping the Long Lists. To this end, it was agreed that the numerical six digit codes of the European Paediatric Cardiac Code would form the backbone of this process of mapping. They would also be used as a structure on which to hang other coding systems that are in use globally, whatever the language.

Prior to the meeting in Toronto, and over the following year, a core group of individuals were involved in systematically scrutinising the mapping of the Short Lists so as to highlight imperfections and areas for debate. The resultant bidirectional crossmap is featured in this Supplement. It should be noted that only a full crossmap was possible of the European Paediatric Cardiac Code Short List to the Short List of the International Congenital Heart Surgery Nomenclature and Database Project. The reasons why the reverse map was not tenable are detailed in the report from the core group involved in this crossmapping project, which appears in the Supplement to this issue of Cardiology in the Young. ${ }^{12}$

\section{The European Paediatric Cardiac Code - the first revision}

Apart from a few minor typographical changes, and numerous minor changes to individual terms to enhance consistency and correctness throughout the system, the following major revisions have been incorporated:

- The addition of 166 new codes to the Short List, mostly as a result of incorporating terms from the International Congenital Heart Surgery Nomenclature and Database Project minimal dataset, which were not exactly represented previously in the European Code. Many of these were higher order generic terms and additional procedures. There were also many additions which took into account advances in procedural rechniques, such as Arterial and atrial switch procedures (double switch), changes in diagnostic nomenclature, such as in the sections devored to pulmonary vascular disease and arrhythmias, and recognition of the changing needs of individual groups, for example, in the sections concerned with fetal diagnoses and arrhythmias.

- The addition of 152 codes from the Long List to the Short List. This "promotion" was the result of requests from many users whose needs have been better recognised since the first publication. This was also required in order to produce a full and exact crossmap between the European Code and each term in the Short List of the Congenital Heart Surgery Project.

- The arrhythmia sections have been completely rewritten to establish a structure much more mechanistic in approach, as requested and now approved by representatives of the appropriate Working Group of the Association for European Paediatric Cardiology. This included the loss of one code from the system which was considered obsolete (Lown Ganong Levine syndrome, 11.07.13).

- The high level generic and "collective" terms previously having the expression "unspecified" appended after the term have had this addition deleted. The coding committee judged that the generic term on its own was self explanatory without the need for this or other clarifying nomenclature, such as "not otherwise specified", being affixed. The "collective terms" will now also be identified in both lists by additional symbols in an additional column ( $\mathrm{cl}$, c2 - see below).

A more detailed breakdown of the revisions and changes in the Short List is given below, whilst these details for the Long List and how it functions are documented in a separate article in the accompanying Supplement. The entire European Paediatric Cardiac Code is fully endorsed again by the Council of the Association for European Paediatric Cardiology, which continues to own the copyright to the system, by the Working Groups for Fetal Echocardiography and Dysrhythmia, as well as the Working Group for adult congenital heart disease of the European Society of Cardiology.

\section{The European Paediatric Cardiac Code Short List}

The revised Short List now has 956 terms with numerical codes, an increase of 315 since the last publication. It remains derived directly from the Long List, with the same structure, including the major division between diagnostic and procedural codes. The structure has now been altered to give two extra columns. The first column now documents those items in the List which are high level generic "collective" terms (" $\mathrm{cl}$ "), and those which are lower level collective 
terms ("c2"). Those without such a designation are primary terms in their own right without subcategories in the Long List. The next columns are unchanged, with the term itself in the second column, the six digit numerical code in the third column, and the fourth through seventh columns committed to the maps to the 9 th and 10th revisions of the International Classification of Diseases ("ICD-9" and "ICD-10"), respectively. The new eighth column documents the crossmap, where possible, to the minimal data set of the International Congenital Heart Surgery Nomenclature and Database Project.

In the reverse listing, the less comprehensive Short List of the Congenital Heart Surgery Nomenclature maps to the European Code. The format of Appendixes IIIa, IIIb, IV, V, and VI is identical to that of the supplement of the Annals of Thoracic Surgery, ${ }^{9}$ representing Non-cardiac diagnoses; General pre-operative risk factors; Diagnoses; Procedures; and Complications, respectively. The first column consists of this term, and is followed by the equivalent term within the European Paediatric Cardiac Code, the six digit numerical code, and then the ninth and tenth versions of the International Classification, in the second through seventh columns, respectively. Details of this list, and the crossmapping process, are in the accompanying article within the Supplement of this issue of Cardiology in the Young. ${ }^{12}$

Otherwise, the structure of the European Paediatric Cardiac Code Short List is little changed. The concept remains that the Short List is a synopsis of the Long List, consisting only of those terms considered to be particularly useful for routine clinical practice, along with "collective" terms to ensure it remains representational of the Long List. Thus, the remaining terms of the Long List within a particular section are covered by this "collective term". For example, there are 38 possible terms for congenital anomalies of the tricuspid valve, with a further 14 qualifying terms for additional detail. These were reduced to a single collective term, tricuspid valvar abnormality, and six specific items: tricuspid atresia, tricuspid regurgitation-congenital, straddling tricuspid valve, tricuspid valvar dysplasia (mucoid thickening), tricuspid stenosis and Ebstein's malformation of tricuspid valve. If the individual diagnosis in question corresponds to a detailed term, then this must be used, whilst the collective term is reserved for all the other diagnoses. As before, the collective terms, or primary terms, are left marginalized in column two, with indentation of the lower hierarchical terms below the related collective term.

There are now eight subgroups to the diagnostic section of the European code Short List of 531 terms:

- Diagnostic congenital and generic cardiac codes, arranged in a sequential segmental format (188 items).
- Diagnostic hereditary, fetal, neonatal and noncardiac codes, including congenital abnormalities associated with cardiac diseases (62 items).

- Diagnostic codes for acquired and post-procedural abnormalities or disorders (173 items).

- Diagnostic codes for disturbances of thythm and conduction (46 items).

- Diagnostic codes for lung and thoracic disease (18 items).

- Diagnostic codes for symptoms and signs (10 items).

- Diagnostic codes for Preoperative Risk Factors (17 items).

- Diagnostic methods (15 items).

The additional penultimate three sections are additions to the Short List to enhance its use as a database for therapeutic procedures, and to aid the day-to-day service of the paediatric cardiologist. In addition are added two Qualifier terms, diagnosis uncertain and status: post-procedure, that can be appended to any term. These were requested as enhancements to database processing and validation.

The therapeutic section with 425 terms has six subsections:

- Cardiac surgical procedures (210 items).

- Arrhythmia, transplant and miscellaneous cardiac procedures (38 items).

- Post-operative and cardiac support procedures (18 items).

- Thorax and lung procedures (26 items).

- Transcatheter procedures (51 items and 1 duplicate from diagnostic methods).

- Procedure related complications (82 items).

It is important to emphasise again that the Short List, particularly now with its greater length, clearly works best as a computer based system, embedded in a database for the collection of information on the diagnosis, intervention and outcome of individual patients. The Short List itself can then be tailored to the needs of the individual clinician, department or institution, only to include relevant areas of interest. As before, it is hoped that such software could then link into a word processing package to produce letters or inpatient summaries, as well as sending off the correct crossmapped codes to the appropriate department for central returns. It should be possible to remove any sight of the numerical codes themselves to aid user friendliness, although a series of defaults should be incorporated to ensure that the chosen items are pathologically valid. For instance, the four terms possible for Double outlet $R V$ are not compatible with the term Discordant VA connections. If a paper based system must be used, then it is advised that particular areas of interest from the Short List should be highlighted, and transferred onto a form of one or 
two sheets to co-exist with a chosen minimal dataset of patient demographics, dates, details of interventions, and outcomes. The dataset as a whole would then be entered onto a computer with the use of diagnostic and procedural key words, immediately accessing the correct code and crossmap required. An example of the multi-level use of the European Paediatric Cardiac coding system is given in the Appendix.

\section{Crossmapping to the 9th and 10th revisions of the International Classification of Disease}

Despite the inadequacies of the International Classification, many central and governmental authorities still require data on the work of physicians and surgeons to be returned using this coding system. In Europe and North America there remain many countries using the 9th revision of the Classification, despite its age, being produced in 1977, and despite its deficiencies and the recommendation of the World Health Organisation to move onto the 10th revision. The crossmap to both revisions, therefore, has been retained. In the United Kingdom, the 9th revision is a four digit code. The fifth digit available in some countries has not been added in anticipation that the 10 th revision will eventually fully supersede the 9 th revision. The detail of how the crossmap between the International Classifications and the European Paediatric Cardiac Code was created has been previously documented. ${ }^{1}$ Apart from minor adjustments to the mapping, there are only two areas where an additional explanation is required. The first is with respect to generic high level terms which refer to lesions which may be either acquired or congenital, such as Mitral stenosis. In the current revision, several of these terms have been added in response to the mapping with the Congenital Heart Surgery Nomenclature and Database Project. In the 10 th revision of the International Classification, items deemed congenital are in Chapter XVII, "Congenital malformations, deformations and chromosomal abnormalities", and are given the prefix $\mathrm{Q}$, whilst acquired lesions are in the Chapter IX, "Diseases of the circulatory system", with the prefix I. This would seem straight forward, but there are arguable exceptions, such as Mitral valvar prolapse being designated as acquired (I34.1), and Eisenmenger's Syndrome being designated as a congenital cardiac septal malformation $(\mathrm{Q} 21.8)$, rather than the more widely accepted designation as an acquired subrype of established pulmonary vascular disease. The latter remains under discussion for change with the World Health Organisation after it was put forward through us to the central coding authority of the United Kingdom. In the current case, it has been elected to place these higher level generic terms under the congenital banner, in keeping with the underlying theme of the European Code, namely congenital heart disease. Thus, the generic term Mitral stenosis has been coded as Q23.2.

The other area of debate was with respect to antenatally diagnosed cardiac abnormalities, where the International Classification has no appropriate codes. This is an important area, as antenatally diagnosed conditions may lead to termination of pregnancy. Technically, one cannot code any details of a congenital abnormality until after the birth of the child. In the 10th revision of the Classification, Chapter XV covers "Pregnancy, childbirth and the puerperium", with items given the prefix $\mathrm{O}$, while Chapter XVI deals with "Certain conditions originating in the perinatal period", these items given the prefix P. In the previous version of the European Code, there was an assumption that Chapter XVI would also cover fetal abnormalities so that most antenatally diagnosed fetal abnormalities, such as Fetal unexplained right heart dominance, were given the code P96.8 - Otber specified conditions originating in the perinatal period with Z36.3Antenatal screening for malformations using ultrasound and otber physical methods or adding a $\mathrm{Q}$ code detailing the congenital anomaly found. After discussion with the central coding authority of the United Kingdom, it is now clear that this was incorrect. Any antenatally diagnosed congenital abnormality must use Chapter XV, specifically $\mathrm{O} 28.3$ - Abnormal ultrasonic finding on antenatally screening of mother as the sole code, despite the reservations above. Unfortunately, the addition of a $\mathrm{Q}$ code for a congenital cardiac lesion would automatically be linked to the mother. The map has, therefore, been adjusted accordingly.

\section{The future}

After the publication of the complete European Paediatric Cardiac Code in 2000, it was expected that the system would need monitoring and updating regularly to incorporate advances in paediatric cardiology and therapeutic procedures, as well as consideration of requests from individuals for improvements to the system and correction of errors that inevitably would become apparent. The current publication is the next stage in this process, incorporating these adjustments and changes, as well as providing a map of the Long to Shorr Lists. The European Code has proved popular across Europe, and is increasingly being used as the standard for multicentric national and international co-operative audit projects, comparing outcomes of both mortality and morbidity, inclusive of case-mix and stratification of risk. The Association for European Paediatric Cardiology continues to hope that other European national bodies concerned with paediatric cardiology will examine the European Paediatric Cardiac Coding System with a view to recommending its use by individual units, or cross linking their own 
systems to it. It is also hoped that translations of the terms into further languages will continue, to aid in the day-to-day use of the system throughout Europe and elsewhere.

It is a measure of the importance of the European code that its underlying numerical structure has been adopted by the global community of paediatric cardiologists and cardiac surgeons as the basis for developing a unified coding system for our speciality. The broader goal of achieving a transglobal international coding and classification system, using mutually exclusive and unambivalent terms, has therefore begun with the eventual aim of establishing a system for effective clinical governance and best practice in our speciality. The use of such a system to audit and monitor the outcome of surgical and medical care in paediatric cardiological practice is increasingly likely to become mandatory in many countries, as the differing outcomes between units are highlighted by "league tables" published by governments and the media. Furthermore, the consequent increased awareness of patients and their families of the variability of outcome between units, and the use of web based information, with a concomitant rise in their expectations, adds further urgency to establishing a valid and truly representative system of audit.

Current technologies for communication have already enabled the establishment of an international paediatric cardiac surgical database using the coding system of the Congenital Heart Surgery Nomenclature and Database Project. ${ }^{10}$ The final global coding system, which is now being developed, should also allow such interactions across the whole speciality, examining mortality and detailed morbidity, such that the outcome of individual centres can be compared to the relevant international gold standard. Appropriate practical advice could then be given where needed, perhaps by televisual links, to improve the outlook of patients at individual institutions throughout the world. This would continue to place paediatric cardiology at the forefront of medical practice in terms of establishing and maintaining valid international standards of best care and outcome for patients of all ages with cardiac disease.

\section{Website}

The Short and Long Lists are available for free download from the web page of the Association for European Paediatric Cardiology: www.aepc.org

\section{References}

1. Franklin RCG, Anderson RH, Daniëls O, et al. The European Paediatric Cardiac Code. Cardiol Young 1999; 9: 633-657.
2. Association for European Paediatric Cardiology. The European Paediatric Cardiac Code. Cardiol Young 2000; 10 (Suppl. 1): 1-146.

3. The Bristol Royal Infirmary Inquiry. The inquiry into the management of care of children receiving complex heart surgery at the Bristol Royal Infirmary. www.bristol-inquiry.org.uk.

4. Smith R. All changed, changed utterly. Brit Med J 1998; 316 : 1917-1918.

5. Spiegelhalter DJ. Mortality and volume of cases in paediatric cardiac surgery: recrospective study based on routinely collected data. Brit Med J 2002; 324: 261-264.

6. Aylin P, Alves B, Best N, et al. Comparison of UK paediatric cardiac surgical performance by routinely collected data 1984-96: was Bristol an outlier? Lancer 2001 ; 358: 181-187.

7. Mavroudis $\mathbf{C}$, Jacobs JP. Congenital heart disease outcome anaysis Methodology and rationale. J Thor Cardiovasc Surg 2002; 123: 6-7.

8. Bonnet D, Coltri A, Butera $G$, et al. Detection of transposition of the great arteries in fetuses reduces neonatal morbidity and mortality. Circulation 1999; 99: 916-918.

9. Congenital Heart Surgery Nomenclature and Database Project. Ann Thorac Surg 2000; 69(4) (Suppl. 1): 1-372.

10. Maruszewski B, Lacour-Gayet F, Elliott MJ, et al. Congenital Heart Surgery Nomenclature and Database Project: update and proposed data harvest. Eur J Cardiothor Surg 2002; 21 : $47-49$.

11. Béland M, Jacobs JP, Tchervenkov CI, Franklin RCG. The International Nomenclature Project for Paediatric and Congenital Heart Disease. Report from the Executive of the International Working Group for Mapping and Coding of Nomenclatures for Paediatric and Congenital Heart Disease. Cardiol Young 2002; 12: $425-430$.

12. Franklin RCG, Jacobs JP, Tchervenkov CI, Béland $M$. Bidirectional Crossmap of the Short Lists of the European Paediatric Cardiac Code and the International Congenital Heart Surgery Nomenclature and Database Project. Report from the Executive of The International Working Group for Mapping and Coding of Nomenclatures for Paediatric and Congenical Heart Disease. Cardiol Young 2002; 12 (Suppl. 2): 18-22.

\section{Appendix: An example of the application of the European paediatric cardiac code}

Systems for the description of individual cases of congenital heart disease, their corresponding procedures and later follow-up, increasingly have to take into account the varying specific demands of the user. The broad spectrum of such a comprehensive coding system has to extend from its use in scientific and clinical cardiological databases, to the prescriptions of state-controlled quality assurance programs, and to its use for reimbursement calculations by health insurance companies. Inevitably, this will entail the application of several different links or crossmaps to other systems. Equally, having a well-defined underlying robust numerical coding structure allows the generation of reproducible lists which are comparable between different paediatric cardiac centres, both nationally and internationally.

As an example of the multifaceted potential of the system, a fictitious case of tetralogy of Fallot has been used. In this case, the hierarchical system naturally falls in to five main divisions (Table 1), which correspond 
Table 1. Example of possible clinical structure for use of the European Paediatric Cardiac Code.

1. Pre-procedural cardiac diagnoses

1.1 Primary cardiac diagnosis

mandatory

1.2 Diagnostic specification: morphological

1.3 Diagnostic specification: hemodynamic

1.4 Diagnostic specification: functional

1.5 Additional cardiac diagnoses

optional

optional

oprional

on demand

2. Relevant non-cardiac diagnoses

2.1 Pre-operative risk faccors

mandatory

2.2 Syndromal or chromosomal factors

2.3 Non-cardiac associations or diagnoses

2.4 Patients past or family history related to heart disease

mandatory

optional

optional

3. Procedures performed (surgical or interventional)

3.1 Primary procedural description mandatory

3.2 Procedural specification : procedural status optional

3.3 Procedural specification : procedura optional category

3.4 Procedural specification : procedural details optional

3.5 Additional procedures

on demand

4. Post-procedural cardiac and non-cardiac complications

4.1 Primary post-procedural cardiac

mandatory complication

4.2 Specifics and sequelae of cardiac

optional complication

4.3 Primary non-cardiac complication

4.4 Specifics and sequelae of non-cardiac complication

4.4 Additional complications

optional

optional

on demand

5. Post-procedural longer term residual and acquired abnormalities

5.1 Primary residual abnormalicy

5.2 Details of residual abnormality

5.3 Primary late acquired abnormality

5.4 Details of late acquired abnormality

5.5 Additional late abnormalities

mandatory

optional

mandatory

optional

on demand

to the usual clinical course of the patient:

- Pre-procedural cardiac diagnoses.

- Relevant non-cardiac diagnoses.

- Procedures performed, be they surgical or transcatheter.

- Post-procedural cardiac and extracardiac complications.

- Post-procedural longer term residual and acquired abnormalities during follow-up.

Each division can then be arbitrarily subdivided into further sections hierarchically arranged depending upon the degree of detail needed, as in the example of tricuspid valve abnormalities given in the
Table 2. Example of clinical use of coding system for Tetralogy of Fallot. Comprehensive listing for detailed research and/or audit.

1. Pre-procedural cardiac diagnoses

\begin{tabular}{|c|c|c|c|}
\hline 1.1 & Tetralogy of Fallot & 01.01 .01 & mandatory \\
\hline 1.2 .1 & Right aortic arch & 09.28 .15 & optional \\
\hline 1.2 .2 & Pulmonary arterial hypoplasia & 09.10 .11 & optional \\
\hline 3 & $\begin{array}{l}\text { Diagnostic } \\
\text { specification: hemodynamic }\end{array}$ & & optional \\
\hline 4 & Hypoxemic spells & 10.17 .12 & optional \\
\hline 5 & $\begin{array}{l}\text { Left SVC persisting to } \\
\text { coronary sinus }\end{array}$ & 04.01 .01 & on demand \\
\hline
\end{tabular}

2. Relevant non-cardiac diagnoses

$\begin{array}{llll}2.1 & \text { Pre-procedural acidosis } & 10.20 .05 & \text { mandatory } \\ 2.2 & \text { 22q11 microdeletion - } & 14.01 .21 & \text { mandatory } \\ & \text { CATCH 22 } & 14.04 .12 & \text { optional } \\ 2.3 & \text { Cleft lip/palate } & 10.23 .01 & \text { optional } \\ 2.4 & \text { Family history of congenital } & & \end{array}$

3. Procedures performed (surgical or interventional)
3.1 Tetralogy of Fallot repai
12.26.01 mandatory
3.2 First operation
(12.43.01) optional
3.3 Cardiopulmonary bypass used 12.00 .90 optional during procedure
3.4 RV to pulmonary artery $\quad 12.36 .01 \quad$ optional conduit construction
3.4.1 - Using pulmonary valve homograft
3.5 Ligation of left SVC
(Q5.96.02) optional
(12.00.37) on demand

4. Post-procedural cardiac and non-cardiac complications

4.1 Post-procedural low cardiac 15.00 .03 mandatory output

4.2 Capillary leak syndrome $\quad 15.80 .06$ optional

4.3 Pleural effusion requiring $\quad 15.80 .61$ optional drainage

4.3.1 Renal failure requiring $\quad 15.82 .06 \quad$ optional temporary dialysis

4.3.2 Instigation of total parenteral (12.32.75) optional nutrition (TPN)

4.4 Delayed closure of sternum $\quad 12.65 .60$ on demand

5. Post-procedural longer term residual and acquired abnormalities

$\begin{array}{llll}5.1 & \text { Residual VSD } & 15.22 .02 & \text { mandatory } \\ 5.2 & \text { Qp:Qs }=<1.5: 1 & (\mathrm{Q} 1.60 .22) & \text { optional } \\ 5.3 & \text { Cardiac conduit failure } & 15.55 .16 & \text { mandatory } \\ 5.4 & \begin{array}{l}\text { Distal cardiac conduit } \\ \text { anastomosis stenosis }\end{array} & (15.55 .04) & \text { optional } \\ 5.4 .1 & \text { - moderate-severe } & (\mathrm{Q} 1.40 .04) & \begin{array}{l}\text { optional } \\ \text { on demand }\end{array} \\ 5.5 & \text { Additional late abnormalities } & & \end{array}$

Numerical codes in parentheses indicate those taken from the European Paediatric Cardiac Code Long List.

body of the text above. Still further additional detail can be coded if the Long List is appended to the system so that the user can "dip" into it as required, for example, to add haemodynamic or anatomical qualifier terms. The highest item in the section would, therefore, often be a "collective" or generic term used 
Table 3. Example of clinical use of coding system for Tetralogy of Fallot. Shortened listing for central returns and/or audit.

List of mandatory primary diagnostic and therapeutic items

1. Pre-procedural cardiac diagnosis

- Tetralogy of Fallot

01.01 .01 mandatory

2. Relevant non-cardiac diagnosis

- 22q11 microdeletion- CATCH $22 \quad 14.01 .21$ mandatory

- Pre-procedural acidosis 10.20 .05 mandatory

3. Procedures performed, surgical or interventional

- Tetralogy of Fallot repair

12.26.01 mandatory

4. Post-procedural complications

- Post-procedural low cardiac output 15.00 .03 mandatory

5. Post-procedural residual and long term acquired abnormalities

- Residual VSD $\quad 15.22 .02$ mandatory

- Cardiac conduit failure

15.55.16 mandatory

The list exclusively includes Short List codes, as illustrated by the use only of mandatory higher level items.

for the individual patient in that division, but which would also correspond to the primary diagnostic or surgical code for audit purposes. These high level items would therefore be exclusively derived from the Short List, with additional detail from both Short and Long lists, as desired. Additional subdivisions can, of course, be created, depending upon the needs of the individual study or unit. Additional linkages to other systems such as the International Classification system or the International Congenital Heart Surgery Nomenclature and Database Project "minimal data set" Short List is then a simple software matter. The latter system can at present only be cross linked at the level of the European Paediatric Cardiac Code Short List.

As an example of this system in operation, Table 2 illustrates a fictitious patient with tetralogy of Fallot, giving a reasonably comprehensive listing of the clinical history of the patient. The layour is as shown in Table 1, with some areas of expansion to add additional detail, and some areas left without data where not relevant to the individual. This much detail could then be fed into a database for detailed research analysis along with the patient demographics, dates and delineated outcomes.

Table 3 then illustrates how the data can be compressed to show only strictly mandatory items, as required for central returns or national audit using a "minimal data set". Any combination of data can evidently be drawn from the comprehensive listing to enable more detailed studies in the future. 